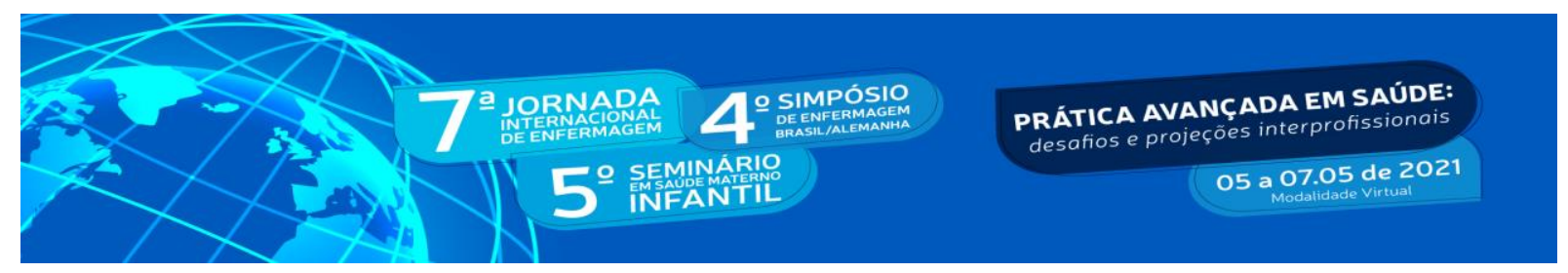

DOI: http://doi.org/10.48195/jie2021-095

\title{
Transtornos traumáticos cumulativos em profissionais de Enfermagem: revisão integrativa das estratégias protetoras ${ }^{1}$
}

\author{
Hilari Silva da Silva²; Julia Machado da Silva ${ }^{3}$; Rielle Herrera Brandli" Talita de \\ Carvalho Leal ${ }^{5}$; Leticia Silveira Cardoso ${ }^{6}$
}

\begin{abstract}
RESUMO
Transtornos traumáticos cumulativos são patologias que afetam estruturas corpóreas, nas quais a dor está presente. Possui origens variadas ocasionando presenteísmo e absenteísmo. Trata-se de uma revisão integrativa realizada com o objetivo de conhecer estratégias protetoras para os transtornos traumáticos cumulativos em profissionais de enfermagem. Realizada na Biblioteca Virtual de Saúde, a partir do protocolo PRISMA. Foram analisados 28 artigos, destes 50\% ressaltaram a educação preventiva como medida para minimizar os transtornos, 39,2\% indicaram comunicação interpessoal, 32,1\% recursos materiais, $25 \%$ relacionou ao aumento do tônus muscular. Compreende que os transtornos traumáticos estão relacionados a fatores organizacionais e ergonômicos deficitários. Cumpriu-se trazer estratégias que minimizem o desenvolvimento dos transtornos, ressaltar a importância da instituição investir nas medidas de promoção, proteção e tratamentos, visando o bem estar de seus trabalhadores e a qualidade dos serviços prestados.
\end{abstract}

Palavras-chave: Saúde do trabalhador; Presenteísmo; Absenteísmo; Estratégias protetoras; Profissionais de enfermagem.

\begin{abstract}
Cumulative traumatic disorders are pathologies that affect bodily structures, in which pain is present. It has varied origins causing presenteeism and absenteeism. It is an integrative review carried out with the objective of knowing protective strategies for cumulative traumatic disorders in nursing professionals. Held in the Virtual Health Library, using the PRISMA protocol. 28 articles were analyzed, of these 50\% emphasized preventive education as a measure to minimize the disorders, $39.2 \%$ indicated interpersonal communication, $32.1 \%$ material resources, $25 \%$ related to the increase in muscle tone. He understands that traumatic disorders are related to deficient organizational and ergonomic factors. It was necessary to bring strategies that minimize the development of disorders, highlighting the importance of the

\footnotetext{
1 Artigo Científico do tipo Revisão de Literatura, Universidade Federal do Pampa - UNIPAMPA.

2 Estudante do Curso de Enfermagem. Universidade Federal do Pampa - UNIPAMPA. E-mail:

hilaris.ferreira397@gmail.com

3 Estudante do Curso de Enfermagem. Universidade Federal do Pampa - UNIPAMPA. E-mail:

juliamachadosilva49@gmail.com

4 Estudante do Curso de Enfermagem. Universidade Federal do Pampa - UNIPAMPA. E-mail:

herrerarielle@gmail.com

5 Estudante do Curso de Enfermagem. Universidade Federal do Pampa - UNIPAMPA. E-mail:

talitacrvl@gmail.com

6 Orientadora. Dr.a em Enfermagem. Universidade Federal do Pampa - UNIPAMPA. E-mail:

leticiacardoso@unipampa.edu.br
} 


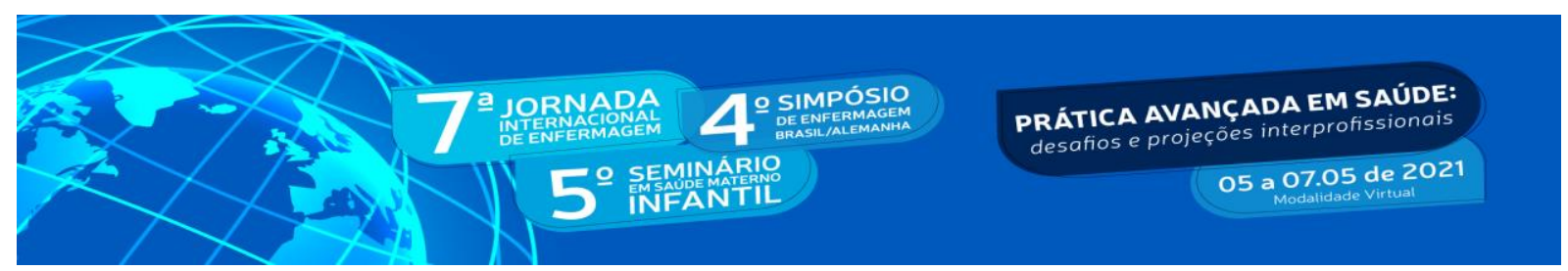

institution to invest in promotion, protection and treatment measures, aiming at the well-being of its workers and the quality of the services provided.

Key Words: Worker's health; Presenteeism; Absenteeism; Protective strategies; Nursing professionals.

\section{INTRODUÇÃO}

Transtornos traumáticos cumulativos são um conjunto de patologias que afetam estruturas musculares e nervosas, de forma isolada ou combinada, nas quais o sintoma de dor está presente. Esses decorrem de condições inflamatórias variadas, podendo ou não ter degeneração de tecidos. Sua origem é multifatorial, envolve hábitos pessoais e situações do ambiente de trabalho, como: extensas jornadas, excesso de peso, várias horas em pé, necessidade de estar sempre alerta, entre outras (ASSUNÇÃO; ABREU, 2017).

$\mathrm{Na}$ contemporaneidade as revelações de desgaste de estruturas do sistema musculoesquelético atingem variadas categorias profissionais. A sua alta prevalência tem sido investigada e relacionada ao estabelecimento de metas, fragmentação e repetitividade das tarefas, flexibilização da jornada de trabalho e controle do tempo em proveito do aumento da produtividade (SOUSA et al., 2016). Entretanto, os transtornos traumáticos cumulativos acometem diretamente o bem estar dos trabalhadores e sua produtividade, promovem o presenteísmo e elevam as taxas de absenteísmo (RIBEIRO et al., 2012). Paralelamente, impactam negativamente a qualidade da assistência à saúde em virtude do reduzido número de profissionais que têm de atender às necessidades dos pacientes e familiares (DALE; DIAS, 2018).

$\mathrm{Na}$ particularidade dos profissionais de enfermagem tem-se vários artigos que confirmam a elevação das taxas referidas, mas escassos são os que indicam estratégias protetoras. Frente ao exposto, esse estudo visa contribuir com o conhecimento científico sobre a implicância que os transtornos traumáticos cumulativos acarretam à qualidade de vida dos profissionais de enfermagem e, principalmente, enfatizar estratégias protetoras.

\section{OBJETIVO}

Conhecer estratégias protetoras para os transtornos traumáticos cumulativos em profissionais de enfermagem. 


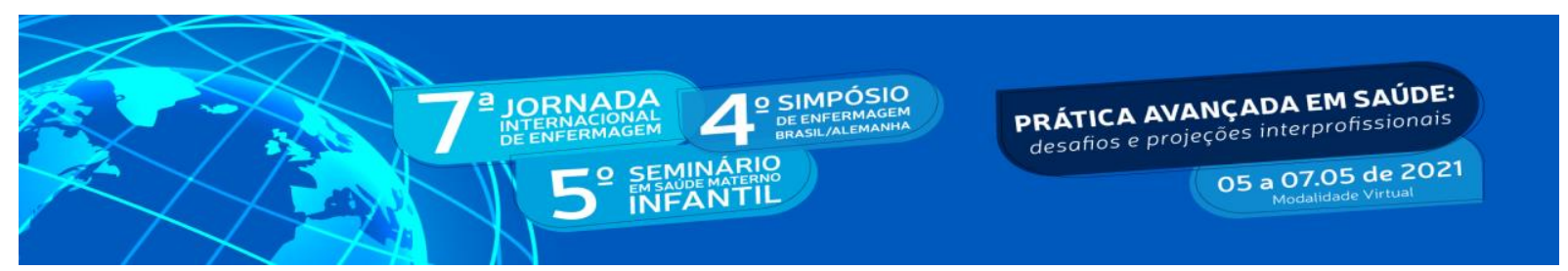

\section{METODOLOGIA}

Trata-se de uma revisão integrativa (SOARES et al., 2014), desenvolvida para responder à questão de pesquisa: "Quais as estratégias protetoras para os transtornos traumáticos cumulativos em profissionais de enfermagem?". Realizou-se a busca pela Biblioteca Virtual de Saúde (BVS), no mês de novembro de 2020, segundo protocolo Systematic Reviews and Meta-Analyses (PRISMA), que consiste em um checklist com o passo a passo de cada etapa, além de um fluxograma (conforme figura 1) de quatro etapas, ajudando a construir a revisão (GALVÃO et al., 2015). Os critérios de inclusão previamente selecionados foram: artigo com acesso completo e livre, nos idiomas de português, inglês ou espanhol. De exclusão: artigos que não apresentassem estratégias de prevenção, promoção ou tratamento de transtornos traumáticos cumulativos e que os participantes não fossem profissionais da enfermagem. Dos 1432 artigos encontrados na base de dados da LILACS, excluíram-se 1416; dos 273 da BDENF, 258; dos 59 da MEDLINE, 57 e dos 19 da IBECS, 18. Obteve-se um corpus analítico de 28 artigos, conforme tabela 1, após sobreposição de bases de dados que produziu a exclusão de seis artigos que estavam duplicados.

Figura 1. Fluxograma da seleção artigos para a revisão integrativa, segundo PRISMA, acerca das estratégias protetoras para os transtornos traumáticos cumulativos em profissionais de enfermagem.

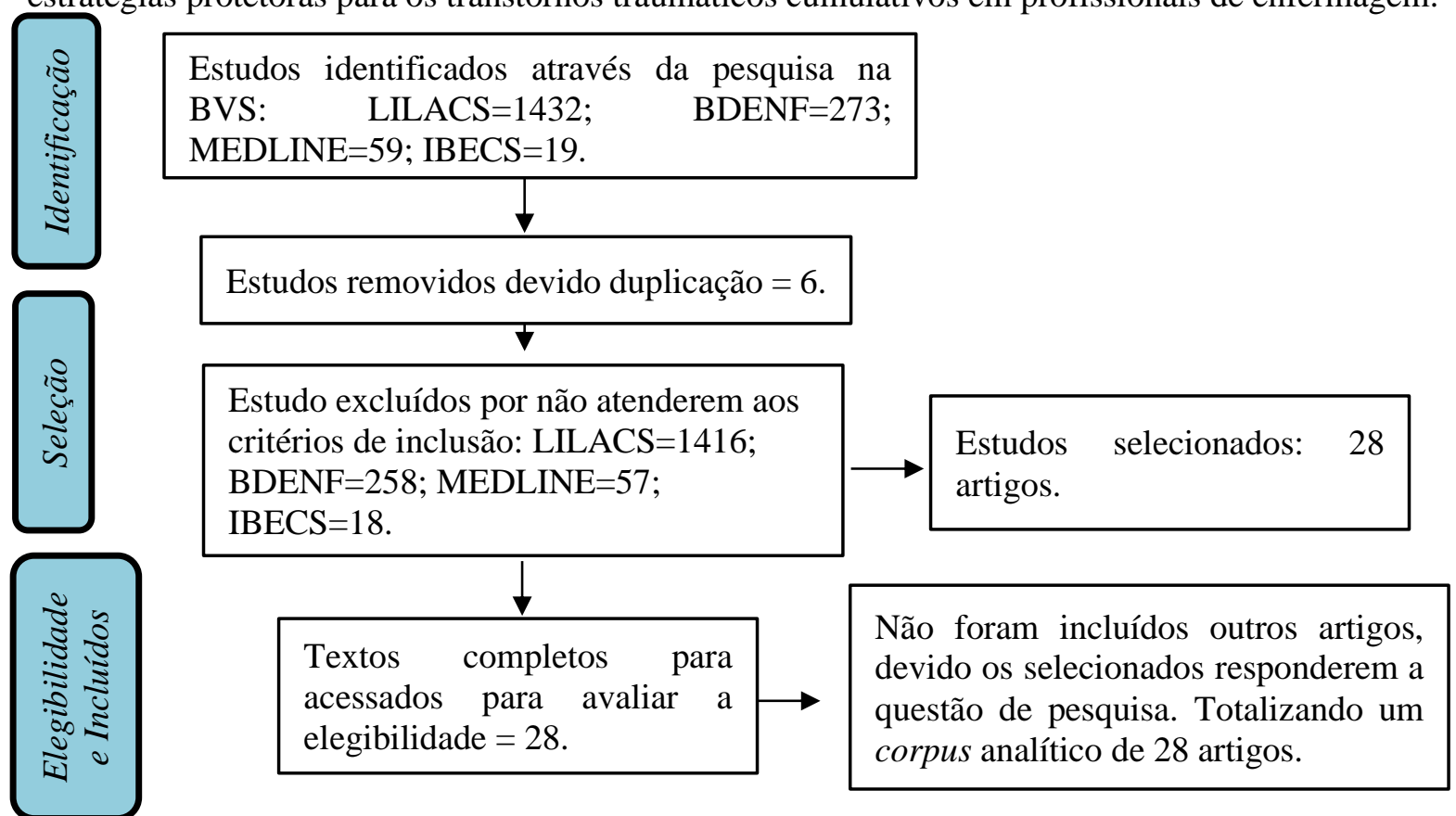

Fonte: autoras. 


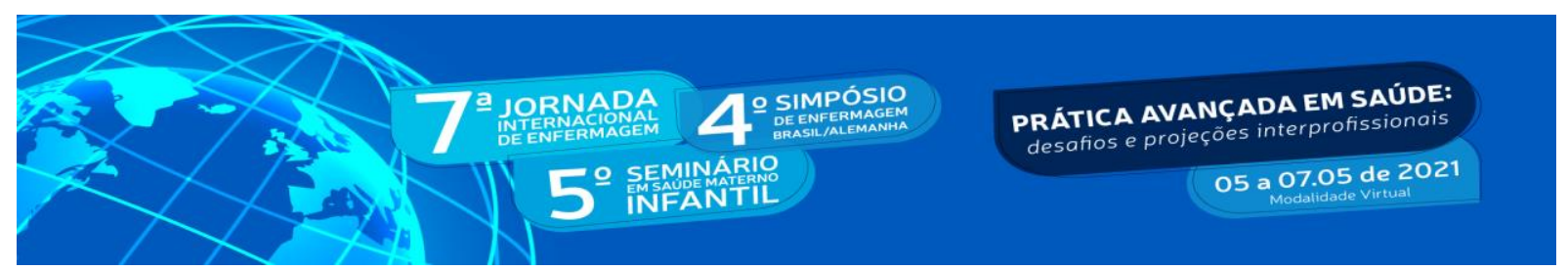

Tabela 1: Corpus Analítico

\begin{tabular}{l|l|l}
\hline $\begin{array}{l}\text { Autores. } \\
\text { Ano de Publicação }\end{array}$ & Título. & Revista. Qualis*. \\
\hline A1: ANTOCHEVIS-DE- & $\begin{array}{l}\text { Trastornos/dolor musculoesquelético en } \\
\text { estudiantes de enfermería de una } \\
\text { universidad comunitaria del sur del } \\
\text { Brasil. }\end{array}$ & Enfermería Global. B1. \\
& Det al., 2017. &
\end{tabular}

BARBOZA et al., 2008.

A3:

CORDIOLI JUNIOR et al., 2020.

A4:

DA SILVA et al., 2019.

A5:

DUARTE et al., 2012.

A6:

FONSECA;

FERNANDES, 2010.

A7:

GALINDO et al., 2016.

A8:

GAMA; TAVARES, 2019.

A9:

HIPOLITO et al., 2015

A10:

LEITE;

MERIGHI;

SILVA, 2007.

A11:

LELIS et al., 2012.
Doenças osteomusculares relacionadas ao trabalho (DORT) e sua associação com a enfermagem ocupacional: [revisão].

Qualidade de vida e sintomas osteomusculares em trabalhadores da atenção primária.

Distúrbio osteomuscular relacionado ao trabalho: identificação dos fatores socioeconômicos e clínicos autorreferidos por trabalhadores de saúde de uma instituição hospitalar do município de Espinosa, Minas Gerais.

Fatores de riscos para distúrbios osteomusculares relacionados ao trabalho - DORT em profissionais de enfermagem.

Fatores associados aos distúrbios musculoesqueléticos em trabalhadoras de enfermagem.

Motivos do absenteísmo em uma equipe de enfermagem ambulatorial.

Development and evaluation of mobile application for the prevention of musculoskeletal risks in nursing work.

A incidência de distúrbios musculoesqueléticos em trabalhadores da equipe de enfermagem em Campos dos Goytacazes.

O cotidiano de trabalhadoras de enfermagem acometidas por distúrbios osteomusculares relacionados ao trabalho (DORT) sob a luz da fenomenologia heideggeriana.

Distúrbios osteomusculares relacionados ao trabalho em profissionais de enfermagem: revisão integrativa da literatura.
Revista Gaúcha de Enfermagem (Online). B1.

Revista Brasileira de Enfermagem. A2.

Revista Rede de cuidados em saúde. B4.

Revista de Pesquisa: Cuidado é Fundamental (Online). B2.

Revista Latino-americana de Enfermagem (Online). A1.

Revista de Enfermagem UFPE On Line. B2.

Texto \& Contexto Enfermagem. A2.

Revista de Pesquisa: Cuidado é Fundamental (Online). B2.

Online Brazilian Journal Of Nursing. B1.

Acta Paulista de Enfermagem. A2. 


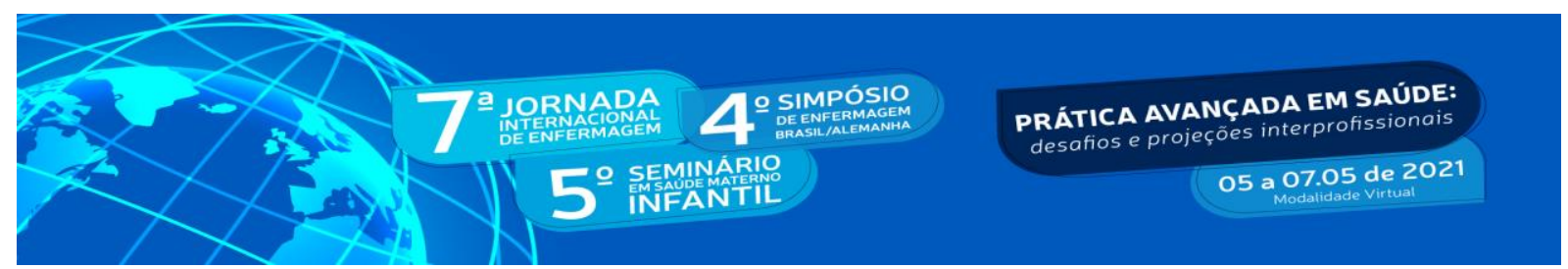

A12:

LOURENÇÃO et al., 2017.

A13:

MACIEL JÚNIOR et al., 2019.

A14:

MAGNAGO et al., 2010.

A15:

MAGNAGO et al, 2012.

A16:

MEDEIROS; SASSO; SCHLINDWEIN, 2018.

A17:

MORAIS et al., 2019.

A18:

PACHECO et al., 2016.

A19:

PORTELA; ROSS, 2015.

A20

ROSA et al., 2008.

A21:

SANTOS; MARZIALE; FELLI, 2018.

A21:

SCHMIDT; DANTAS, 2012.

A23:

SILVA et al., 2017.

A24:

SILVA et al., 2020.

A25:

SOUZA et al., 2020.
Queixas de distúrbios osteomusculares em aprimorandos e aperfeiçoandos atuantes em um hospital de ensino.

Self-reported musculoskeletal disorders by the nursing team in a university hospital.

Aspectos psicossociais do trabalho e distúrbio musculoesquelético em trabalhadores de enfermagem.

Intensidade da dor musculoesquelética e a (in)capacidade para o trabalho na enfermagem.

Results of foot reflexotherapy in acute lower back pain of the nursing team: controlled randomized clinical test.

Musculoskeletal pain in undergraduate health students: prevalence and associated factors.

Prevalência dos sintomas osteomusculares relacionados ao trabalho de enfermagem no âmbito hospitalar.

Distúrbios osteomusculares relacionados ao trabalho (DORT) e sua associação com condições de trabalho da enfermagem.

Incidência de LER/DORT em trabalhadores de enfermagem.

Presenteeism and musculoskeletal symptoms among nursing professionals.

Qualidade de Vida no Trabalho e Distúrbios Osteomusculares Relacionados ao Trabalho entre profissionais de enfermagem.

Presença de Distúrbios Osteomusculares em Enfermeiros de Unidades de Pronto Atendimento.

Distúrbios osteomusculares e ações para reduzir a ocorrência em trabalhadores de enfermagem.

Caracterização dos trabalhadores da enfermagem afastados por distúrbios osteomusculares em hospital universitário.
Revista de Enfermagem UFPE On Line. B4.

Brazilian Journal of Pain. SEM*

Revista Latino-americana de Enfermagem (Online). A1.

Revista Latino-americana de Enfermagem (Online). A1.

Brazilian Journal of Pain. SEM*

Revista da Escola de Enfermagem da USP. A2.

Revista de Enfermagem da UFPI. B4.

Revista de Enfermagem da UFPI. B4.

Acta Scientiarum. Education (Online). A2.

Revista Latino-americana de Enfermagem (Online). A1.

Acta Paulista de Enfermagem.

A2.

Revista de Enfermagem e Atenção à Saúde. B3.

Revista Enfermagem UERJ. B1.

Revista de enfermagem da UFSM. B2. 


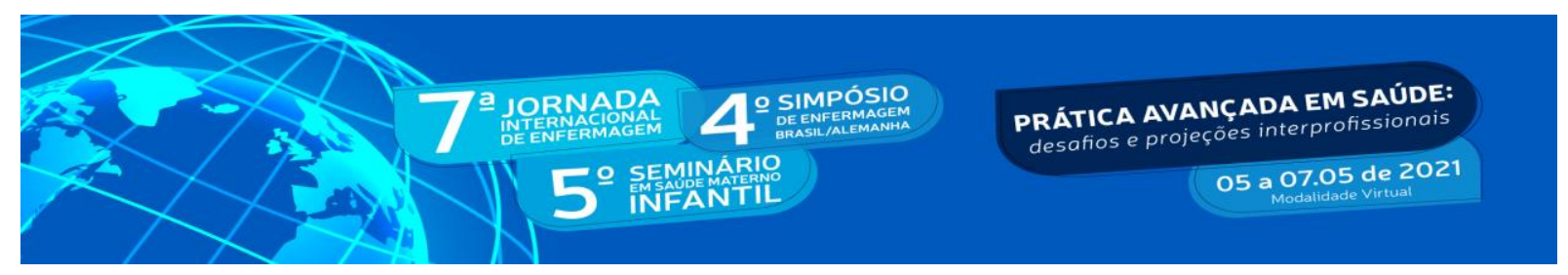

A26:

TINIBU et al., 2010.

A27:

VALENÇA; ALENCAR, 2015.

A28:

VARELA; FERREIRA, 2004.

\begin{tabular}{l|l}
2004. & Salv \\
\hline Total & 28 \\
\hline
\end{tabular}

Distúrbios

musculoesqueléticos

BMC CANCER. A1.

relacionados ao trabalho entre enfermeiras em Ibadan, sudoeste da Nigéria: um estudo transversal.

Distúrbios osteomusculares e o trabalho de técnicos e auxiliares de enfermagem em instituições de idosos.

Perfil das trabalhadoras de enfermagem com diagnóstico de LER/DORT em

Revista Brasileira de Enfermagem. A2.

* Sucupira, 2013-2016.

Dos 111 autores presentes no conjunto dos 28 artigos, 81 são enfermeiros, 9 fisioterapeutas, 8 médicos, 3 psicólogos, 1 educador físico, os demais de outras áreas do conhecimento. A fonte de dados de maior predomínio do corpus foram instituições hospitalares municipais/estaduais/privado com oito cenários (A4; A6; A8; A11; A18; A22; A26; A27), as instituições universitárias com sete cenários (A7; A12; A13; A15; A21; A25; A28), os artigos bibliográficos e integrativos com cinco cenários (A2; A5; A11; A19;A20), dois artigos não informaram o cenário (A10; A17), três em universidades (A1; A14; A27). Por último, as estratégias protetoras em cenários da atenção básica de saúde foram contempladas em um artigo (A3) em unidades clínicas e pronto atendimento com um aparecimento para cada, respectivamente (A1; A23).

Para análise corpus realizou-se a leitura e releitura do conjunto de artigos selecionados para identificar o que se faz indispensável para obtenção de um estudo aprofundado a respeito da temática e para o alcance do objetivo proposto. Para apresentação e discussão dos resultados agruparam-se os dados em duas categorias teórico empíricas: Dor o sinal presente nos Transtornos Traumáticos Cumulativos em Profissionais de Enfermagem e Estratégias Protetoras para os Transtornos Traumáticos Cumulativos em Profissionais de Enfermagem.

Os aspectos éticos e os preceitos de autoria foram respeitados visto que os autores consultados foram citados e referenciados ao longo do estudo. Paralelamente, se dispôs o ano de publicação dos documentos conforme previsto na Lei $\mathrm{n}^{\circ} 9.610$ de 19 de fevereiro de 1998 (BRASIL, 1998). 


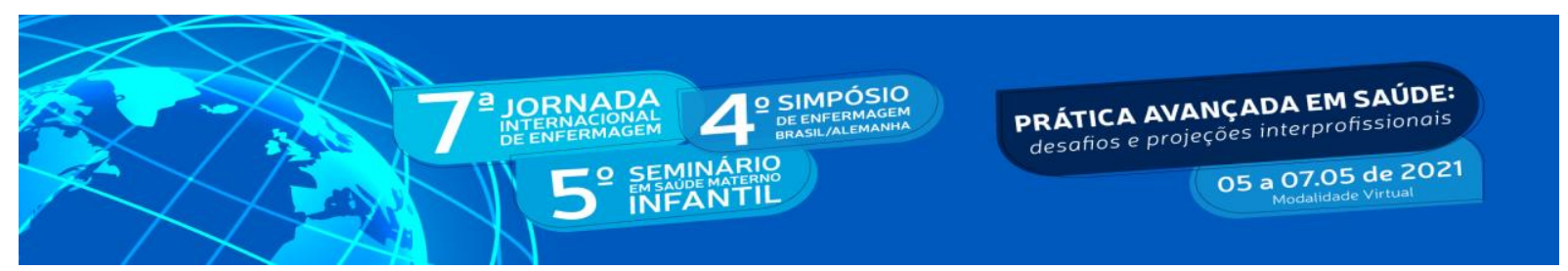

\section{RESULTADOS}

Destaca-se que os dados não são excludentes, ou seja, um artigo compõe mais de uma categoria e, é variável.

\section{Dor o sinal presente nos Transtornos Traumáticos Cumulativos em Profissionais de Enfermagem}

No conjunto de 28 artigos, houve a indicação de dor em 45 relatos. Destes, 21 (75\%) indicaram a lombalgia (A1; A3 a A7; A11 a A13; A15; A17; A19 a A22; A23 a A27), 14 (50\%) a dor nos ombros e cervical (A1; A3; A5; A7; A12 e A13; A17; A19 a A21; A23 a A25; A27), 7 (25\%) nos punhos/mãos, quadril/coxas e joelhos (A2 e A3; A7; A13; A17; A24 e A25), 2 $(7,14 \%)$ tornozelos e cotovelos (A12; A24) e, 1 (3.57\%) tórax (A13). O gênero feminino predominou no corpus investigado, $24(85,7 \%)$ dos 28 analisados.

\section{Estratégias Protetoras para os Transtornos Traumáticos Cumulativos em} Profissionais de Enfermagem.

Dos $28(100 \%)$ artigos, $14(50 \%)$ ressaltam a educação preventiva relacionada à mecânica corporal no desenvolvimento das ações assistenciais (A1 e A2; A5 e A6; A9; A11 e A12; A15; A19; A21 a A23; A26; A28). Outros 11 (39,2\%) indicam a comunicação interpessoal efetiva entre os envolvidos com a assistência à saúde como principal estratégia protetora da equipe de enfermagem (A2 e A3; A7; A11; A14 e A15; A21 a A24; A26). Já 9 (32,1\%) apontam para a disponibilidade de recursos materiais que evitem uso excessivo da força física para assistir os pacientes (A1; A3 e A4; A10; A12 e A13; A16; A20; A28). A ginástica laboral foi referida em 7 (25,0\%) artigos como a possibilidade de ampliar o tônus e a força muscular para o desempenho das ações de assistência (A1; A9; A13; A17; A19; A23 e A22).

A reflexão sobre múltiplos vínculos empregatícios foi mencionada em $6(21,4 \%)$ artigos como fator contributivo para o desgaste profissional da equipe de enfermagem (A7; A9; A11 e A12; A18; A23). E o acesso às tecnologias terapêuticas, mencionadas em 4 (14,2\%) artigos auxiliam a amenizar a dor provocada pelos transtornos traumáticos cumulativos (A8; A11; A14; A25). 


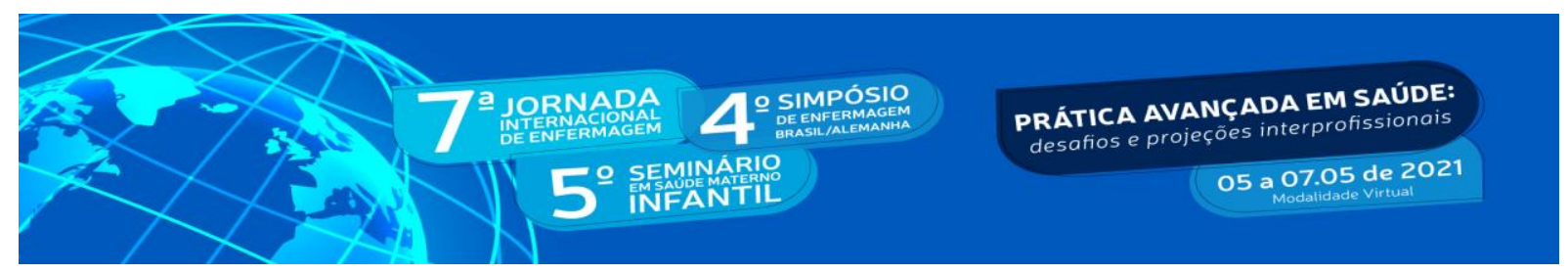

\section{DISCUSSÃO}

Observa-se que a lombalgia é um dos sintomas mais frequentes em profissionais de enfermagem em decorrência de suas ações de trabalho. A cronificação da lombalgia tem entre os fatores causais a realização de excessivas horas de trabalho e o modo errôneo de movimentação corporal nas ações de trabalho. Ela é um dos grandes motivos de absenteísmo e diminuição da qualidade de vida dos profissionais de enfermagem (RAGIEVICZ, 2018).

O absenteísmo muitas vezes oneram o sistema público de seguridade social e o próprio cotidiano de trabalho da equipe a qual este profissional pertence (CARNEIRO; ADJUNTO, 2017). Ele pode ser precedido pelo presenteísmo, no qual o profissional pode estender a outros seus conflitos, comprometendo a saúde dos demais trabalhadores e, em algumas situações, do paciente (VIEIRA et al., 2016).

A questão do predomínio de mulheres na enfermagem, encontrada neste estudo, não representa por si só um dado importante. Entretanto, ao pensar em sua relação com a dor podese inferir a conjugação de ações de trabalho em diferentes cenários para além do profissional (RIBEIRO et al., 2012). Outro aspecto a ser considerado está nas diferenças entre massa muscular, óssea, fraqueza articular e altura em relação ao sexo masculino, já a idade associada a um maior tempo de experiência profissional revelou-se como fator de minimização de transtornos traumáticos cumulativos (RUSCH et al., 2019).

O comprometimento e engajamento da equipe de enfermagem para a implementação de estratégias de amenização dos transtornos traumáticos cumulativos deve estar ao encontro do uso correto dos recursos. A instituição também deve se esforçar na adequação do ambiente de trabalho, tornando-o seguro a todos (CARVALHO et al., 2019). Uma possibilidade para consolidação do referido está na gerência participativa, que viabiliza aos profissionais/trabalhadores se incluírem na busca de soluções aos problemas diagnosticados (LOPES et al., 2019). Reforçando em paralelo sentimentos individuais de identificação com a instituição e as equipes de trabalho (NOVATO; NUNES, 2019).

A implementação da ginástica laboral, como forma de amenizar os transtornos 


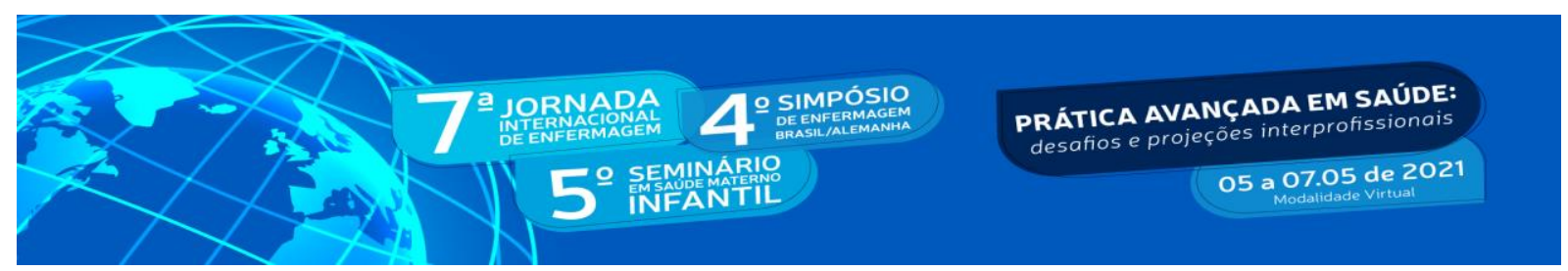

traumáticos cumulativos, tem se mostrado eficaz. Paralelamente, ela influencia beneficamente a realização de exercícios físicos fora da instituição (SANTANA; MENDES, 2019). A estes se acrescentam outras terapias não farmacológicas utilizadas para aliviar dores (ROCHA; FRAZÃO, 2018).

\section{CONCLUSÃO}

A interação interprofissional mediada pelo acesso a informações representou a principal estratégia protetora de transtornos traumáticos cumulativos em profissionais de enfermagem. Esta indicou ainda uma necessidade de expressão de sentimentos de pertencimento e reconhecimento destes profissionais em relação à instituição, demais profissionais e pacientes/sociedade.

Outra constatação relevante está na priorização de estratégias protetoras previamente ao estabelecimento da patologia, a exemplo da ginástica laboral. É importante ressaltar que as estratégias protetoras estão atreladas ao nível de atenção dos serviços de saúde e que os enfermeiros são os principais produtores de estudos com essa temática.

\section{REFERÊNCIAS}

ASSUNÇÃO; A. A.; ABREU, M. N. S. Fatores associados a distúrbios osteomusculares relacionados ao trabalho autorreferidos em adultos brasileiros. Rev Saúde Pública, São Paulo, v. 51, supl. 1:10s, 2017.

BRASIL. Lei $\mathrm{n}^{\circ}$ 9.610, de 19 de fevereiro de 1998. Altera, atualiza e consolida a legislação sobre direitos autorais e dá outras providências. Diário Oficial da República Federativa do Brasil, Brasília, DF, 20 fev. 1998.

CARNEIRO, V.; ADJUTO, R. N. P. Fatores relacionados ao absenteísmo na equipe de enfermagem: uma revisão integrativa. Rev. Administração em Saúde, São Paulo, v. 17, n. 69, 2017.

CARVALHO, D. P., et al. Workloads and burnout of nursing workers. Rev. Bras. Enferm., Brasília, v. 72, n. 6, p. 1435-1441, 2019. 


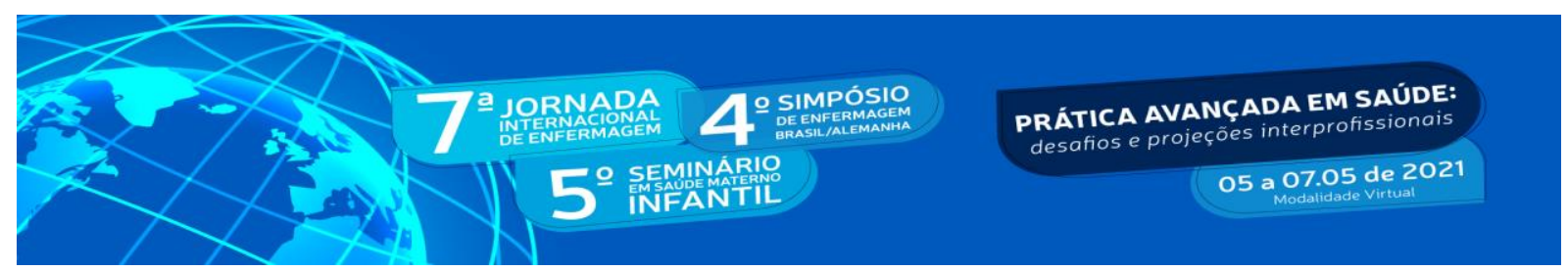

DALE, A. P.; DIAS, M. D. D. A ‘extravagância’ De Trabalhar Doente: O Corpo No Trabalho Em Indivíduos Com Diagnóstico De Ler/Dort. Trab. educ. saúde, Rio de Janeiro, v. 16, n. 1, p. 263-282, 2018.

GALVÃO, T. F., et al. Principais itens para relatar Revisões sistemáticas e Meta-análises: A recomendação PRISMA. Epidemiologia e Serviços de Saúde, Brasília, v. 24, n. 2, p. 16791683, jun. 2015.

LOPES, T. D. Q., et al. Gestão participativa e educação permanente: uma receita para a qualidade do trabalho na atenção básica. Archives of health investigation, Araçatuba, v. 7, jan. 2019.

NOVATO, D.; NUNES, E. C. D. A. As relações interpessoais na enfermagem: influência da liderança na motivação da equipe técnica. Revista Saúde - UNG-Ser, Guarulhos, v. 13, n. 1⁄2, 2019.

RAGIEVICZ, D. A. Lombalgia Ocupacional Em Profissionais Da Enfermagem: Uma Revisão Bibliográfica. Repósito Digital Uri Erechim, Erechim, 2018.

RIBEIRO, N. F., et al. Prevalência de distúrbios osteomusculares relacionados ao trabalho em profissionais de enfermagem. Rev. bras. epidemiol., São Paulo, v. 15, n. 2, p. 429-438, jun. 2012.

ROCHA, L. M. N.; FRAZÃO, R. S. Educação profissional na prevenção de doenças osteomusculares de trabalhadores do processo produtivo. 2018, Dissertação. (Mestrado em Ciências da Educação) - Faculdade de Ciências Sociais, Educação e Administração.

RUSCH, M. H., et al. Dor Lombar Em Profissionais Da Enfermagem Em Um Hospital Do Vale Do Rio Pardo - RS. UNISC, Santa Cruz do Sul, 2019.

SANTANA, K. A.; MENDES C. M. M. Ginástica laboral na prevenção da DORT/LER dos trabalhadores. UNA-SUS, p. 1-13, 2019.

SOARES, C.B., et al. Revisão Integrativa: Conceitos e métodos utilizados na enfermagem. Esc Enferm., São Paulo, v. 48, n. 2, p. 335-345, 2014.

SOUSA, B. V. N., et al. Lesões Por Esforço Repetitivo Em Profissionais De Enfermagem: Revisão Sistemática. Revista Brasileira de Saúde Funcional, Cachoeira, v. 1, n. 3, p. 59, 15 dez. 2016.

VIEIRA, M. L. C., et al. Precarização do trabalho em hospital de ensino e presenteísmo na enfermagem. Rev. Enfermagem Uejr, Rio de Janeiro, v. 24, n. 4, 2016. 